\title{
O COMPONENTE CULTURAL NAS INTERAÇÕES DE TELETANDEM: TEORIAS E REFLEXÕES
}

\author{
EL COMPONENTE CULTURAL EN LAS INTERACCIONES DE TELETANDEM: \\ TEORÍAS Y REFLEXIONES
}

\author{
THE CULTURAL COMPONENT IN TELETANDEM INTERACTIONS: \\ THEORIES AND REFLECTIONS
}

\author{
Giovana MORETTI ${ }^{1}$ \\ Ana Cristina Biondo SALOMÃO ${ }^{2}$
}

RESUMO: O "Teletandem Brasil: línguas estrangeiras para todos" é um projeto desenvolvido pela UNESP com o objetivo de proporcionar a aprendizagem de línguas estrangeiras ao colocar pares de falantes de diferentes línguas em contato síncrono, de forma colaborativa, por meio de recursos de comunicação na Internet (TELLES; VASSALLO, 2006). Fundamentado em pesquisas realizadas anteriormente acerca do componente cultural nesse contexto, este artigo visa apresentar e refletir a respeito dos conceitos teóricos sobre cultura abordados e o modo como podemos observar sua presença na experiência dos participantes, tanto nas sessões de interação quanto nas mediações subsequentes. Podemos concluir que essa modalidade de aprendizagem colaborativa promove a emergência do componente cultural de forma dinâmica entre os participantes como um processo de reflexão sobre sua própria língua e cultura proporcionada pela interação com o outro.

PALAVRAS-CHAVE: Telecolaboração. Línguas estrangeiras. Teletandem. Cultura.

RESUMEN: "Teletandem Brasil: lenguas extranjeras para todos" es un proyecto desarrollado por la UNESP con el objetivo de proporcionar el aprendizaje de lenguas extranjeras al colocar pares de hablantes de diferentes lenguas en contacto síncrono, de forma colaborativa, por medio de recursos de comunicación en Internet (TELLES; VASSALLO, 2006). Fundamentado en investigaciones realizadas anteriormente sobre el componente cultural en ese contexto, este artículo pretende presentar y reflexionar acerca de los conceptos teóricos sobre cultura abordados y como podemos observar su presencia em la experiencia de los participantes, tanto en sesiones de interacción como en las mediaciones posteriores. Podemos concluir que esta forma de aprendizaje colaborativo promueve el surgimiento dinámico del componente cultural entre los participantes como un proceso de reflexión sobre su propio idioma y cultura proporcionado por la interacción con el otro.

PALABRAS CLAVE: Telecolaboración. Lenguas extranjeras. Teletandem. Cultura.

${ }^{1}$ Universidade Estadual Paulista (Unesp), Araraquara - SP - Brasil. Mestranda em Linguística e Língua Portuguesa. ORCID: https://orcid.org/0000-0001-9057-3950. E-mail: giovanamoretti@live.com

${ }^{2}$ Universidade Estadual Paulista (Unesp), Araraquara - SP - Brasil. Professora do Departamento de Letras Modernas. ORCID: https://orcid.org/0000-0002-1531-8551. E-mail: ana.salomao@unesp.br 
ABSTRACT: "Teletandem Brasil: foreign languages for all” is a project developed by UNESP with the aim of providing the learning of foreign languages by placing pairs of speakers of different languages in contact synchronously, in a collaborative way, through communication resources on the Internet (TELLES; VASSALLO, 2006). Based on previous research about the cultural components in this context, this article aims to present and reflect on the theoretical concepts about culture addressed in such research, in order to identify how these concepts apply to the context of Teletandem, and how we can observe their presence in the participants' experience, both in interaction sessions and in subsequent mediations. We can conclude that this form of collaborative learning promotes the emergence of the cultural component dynamically among the participants as a process of reflection on their own language and culture provided by the interaction with the other.

KEYWORDS: Telecollaboration. Foreign languages. Teletandem. Culture.

\section{Introdução}

$\mathrm{Na}$ contemporaneidade, aprender uma língua estrangeira tem sido o objetivo de muitas pessoas por diversas razões, que variam desde a satisfação pessoal por aprender algo novo até as necessidades que surgem devido ao trabalho ou à vida acadêmica. Porém, quando aprendemos um novo idioma, não nos limitamos apenas a aprender regras gramaticais, vocabulário e outras estruturas linguísticas, mas também acabamos nos deparando com outros aspectos que surgem a partir da interação com a nova língua, a âmbitos culturais, históricos e sociais.

Concomitantemente ao aumento do interesse pelo ensino e aprendizagem de um novo idioma, as Tecnologias de Informação e Comunicação (TIC) também avançam rapidamente, proporcionando mais recursos de comunicação síncrona por meio de voz e vídeo aos seus usuários, como as chamadas via Skype, WhatsApp e, mais recentemente, o Zoom. Estes recursos de comunicação têm possibilitado com mais facilidade a interação entre pessoas de diferentes lugares do mundo, mostrando-se de grande proveito para o ensino e a aprendizagem de línguas estrangeiras, como, por exemplo, no projeto "Teletandem Brasil: línguas estrangeiras para todos" (TELLES; VASSALLO, 2006; VASSALLO; TELLES, 2006).

A proposta do Teletandem se baseia na premissa do contexto tandem, palavra do latim utilizada originalmente para designar uma bicicleta de dois lugares na qual as pessoas pedalam juntas e contribuem para a locomoção rumo a um destino comum; no contexto linguístico, o tandem é uma modalidade de aprendizagem colaborativa na qual 
dois falantes de línguas diferentes compartilham conhecimento sobre tais línguas e atuam tanto como aprendizes quanto como tutores.

No Teletandem, os interagentes entram em contato um com o outro por meio de recursos tecnológicos de comunicação, tais como o Skype e outros aplicativos que permitem a troca de mensagens, áudios e vídeos instantaneamente, e dividem o tempo de interação entre as duas línguas alvo. $\mathrm{O}$ ensino e aprendizagem compartilhados entre os interagentes podem se desenvolver tanto em níveis linguísticos quanto culturais, e as novas tecnologias têm sido fortes aliadas para que esse processo ocorra.

A figura a seguir ilustra uma sessão de Teletandem na modalidade institucional, realizada em um laboratório de idiomas nas dependências da universidade. Uma sessão é geralmente composta por uma hora de interação e meia hora de mediação. A interação é o momento em que os parceiros ajudam um ao outro no processo de aprendizagem, pautando-se nos princípios da autonomia, reciprocidade e separação de línguas (TELLES; VASSALLO, 2006). A mediação é uma sessão reflexiva em grupo sobre a interação e a aprendizagem nesse contexto, que ocorre na forma de roda de conversa na meia hora seguinte à interação. No Brasil, os mediadores são alunos de graduação ou pós-graduação, com experiência em Teletandem, que, em sua maioria, conduzem pesquisas nesse contexto. O objetivo é fazer com que o grupo de participantes discuta sobre aspectos relacionados à prática do aluno e reflita juntos acerca das dúvidas, dos problemas encontrados no ensino e aprendizagem de línguas nas sessões de teletandem, questões culturais e possíveis impasses (SALOMÃO, 2011).

Figura 1: Participantes do Teletandem durante interação na Unesp de Araraquara

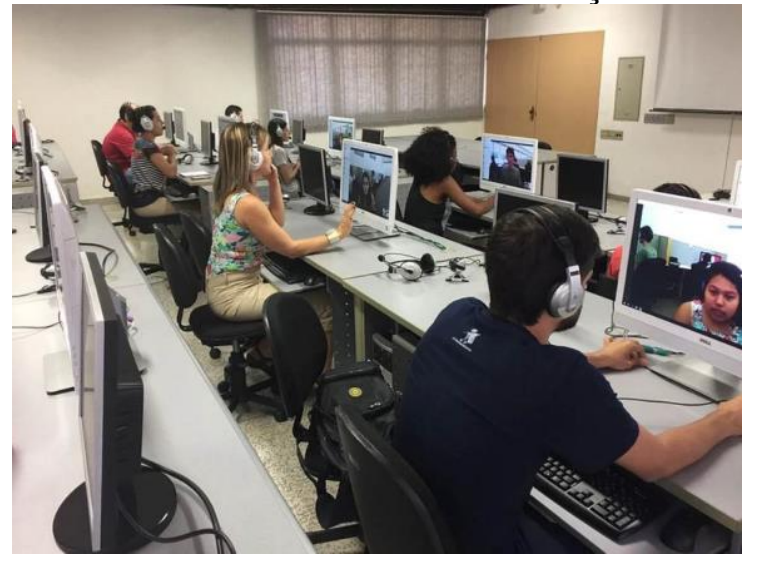

Fonte: http://teletandem.wixsite.com

Salomão (2012) afirma que o ensino de línguas deve ser pensado considerando fluxos culturais, discursivos e linguísticos e também o caráter político das relações entre 
língua, cultura e sociedade, para que assim os aprendentes se tornem capazes de interagir com pessoas que pensem e ajam de forma diferente. Inseridos no contexto do Teletandem, os participantes do projeto entram em contato por meio da telepresença (SALOMÃO, 2015) com contextos culturais e sociais diferentes dos seus e têm a oportunidade de desenvolver sua produção em língua estrangeira por meio da interação.

Considerando que os participantes do projeto são proficientes em diferentes idiomas e vivem em diferentes contextos culturais, espera-se que o aprendizado de tais falantes não se resuma apenas à língua-alvo, mas também se estenda a aspectos sociais, culturais e históricos acerca da realidade do parceiro de interações. Por meio do contato intercultural, os falantes podem encontrar um espaço para refletir sobre - e compreender - as culturas, conforme reitera Zakir (2015). Essa reflexão tende a trazer amplitude para o conhecimento adquirido além do linguístico, indo de encontro a fenômenos como os estereótipos, preconceitos enraizados e relações de poder. Isto posto, a experiência do Teletandem quando pautada por uma abordagem reflexiva pode se ampliar significativamente, trazendo novas perspectivas de mundo e maior compreensão quanto ao nosso lugar na relação com o outro.

Levando em conta as propostas do projeto Teletandem Brasil e as observações e análises feitas em publicações acadêmicas anteriores acerca dos elementos culturais presentes nas interações entre os falantes, buscamos refletir sobre como esses aspectos e teorias foram abordados nestes trabalhos e a quais conclusões podemos chegar com base nesses estudos, indicando encaminhamentos para a prática nesse contexto colaborativo.

\section{Elementos culturais nas interações do Teletandem}

Considerando as propostas do projeto Teletandem, sua aplicação e os três princípios norteadores do tandem propostos por Telles e Vassallo (2006), podemos inferir que o contexto das interações telecolaborativas difere-se do contexto da sala de aula e, portanto, a abordagem dos aspectos culturais que aplicamos aos alunos ocorre de maneira distinta do que os participantes do Teletandem experienciam.

$\mathrm{Na}$ sala de aula, professores geralmente exploram a cultura por meio do que é apresentado nos materiais didáticos, datas comemorativas (St. Patrick's Day, Thanksgiving, etc.), ou até mesmo através da exposição de itens lexicais e seus usos (por exemplo, o uso dos pronomes de tratamentos Miss, Mrs., Ms. e Mr. e suas regularidades ao serem comparados com o uso equivalente na língua portuguesa), entre 
outras abordagens, que geralmente são mais voltadas à discussão sobre a cultura na forma de práticas cotidianas e as possíveis implicações de determinados comportamentos. No Teletandem, por sua vez, a cultura está representada no parceiro, gerando a oportunidade de reflexão pela observação do processo de identificação (ou falta dela) entre os aprendizes que trabalham colaborativamente sem roteiros prévios definidos. Assim, nesse contexto, cultura passa a ser uma possibilidade concreta na interação com o outro.

Durante a telecolaboração, ao entrar em contato direto com o parceiro, é possível que o aprendente se depare com situações muito mais próximas à vida real do que em sala de aula, fugindo de padrões impostos por sistemas educacionais ou métodos, conforme afirma Salomão (2015):

Ao dissociarmos a língua inglesa de uma estrutura de referência nacional, baseada em uma língua estândar e em uma línguacultura padronizada ('inglês americano', 'inglês britânico'), concentrada nos discursos da cultura dominante, procuramos prover aos alunos uma experiência de uso da língua que reflete os possíveis contatos dos aprendizes fora de sala de aula - que não serão somente com pessoas que falam a língua-alvo como primeira língua (SALOMÃO, 2015, p. 384).

A aprendizagem telecolaborativa também se destaca da sala de aula no sentido de que proporciona um ambiente no qual os falantes/aprendentes podem tirar dúvidas e resolver inquietações sobre o que estão aprendendo e a maneira como estão utilizando a língua estrangeira em tempo real, por meio da negociação de significados. Para que os objetivos sejam alcançados, o parceiro de interação precisa estar engajado ao contexto e contribuindo ativamente para o desenvolvimento da interação (RODRIGUES, 2013).

Evangelista e Salomão (2019) afirmam que os interagentes podem não ser capazes de explorar de forma completa o potencial que esse contexto oferece em relação às reflexões possibilitadas pelo encontro com o outro. Nesse sentido, entendemos, como as autoras, que a mediação estabelece um contexto informal de conversa entre mediador e participantes no qual são compartilhadas experiências de ensino e aprendizagem, nível de conforto durante as interações, de que maneira se deu a negociação de sentidos, entre outros pontos pertinentes à ocasião conforme a sessão se desenrola. Desse modo, neste contexto pós-interação, o mediador ocupa papel importante ao conduzir as reflexões necessárias para que os participantes possam problematizar questões interculturais e, a partir disso, desconstruir e/ou ressignificar discursos enraizados sobre a língua e a cultura do outro e até sobre a sua própria. 
Isto posto, a reflexão sobre cultura nesse contexto colaborativo de aprendizagem, tanto durante as interações quanto no momento da mediação, engendra a complexidade de elementos que envolvem identidade e alteridade. Nesse sentido, várias pesquisas têm sido conduzidas com enfoque em tais temáticas, mostrando que há uma gama de possiblidades nas formas de se abordar aspectos culturais na prática telecolaborativa. Assim, nos propusemos a fazer um levantamento sobre a forma como os aspectos culturais têm sido tratados em pesquisas no teletandem a fim de compreender como elas se diferem e/ou complementam para indicar possíveis encaminhamentos para a prática nesse contexto.

\section{Questões culturais no contexto do Teletandem em trabalhos acadêmicos já realizados}

Para refletirmos mais a respeito destas questões culturais no contexto do Teletandem, nos dedicamos à leitura de alguns trabalhos acadêmicos já realizados sobre este tema. Nosso intuito é discutir de que maneira esses trabalhos se assemelham e se diferem em se tratando da abordagem de cultura, seus objetivos e os resultados obtidos por seus autores. Para iniciarmos tal comparação, apresentamos o quadro a seguir, no qual contemplamos os seis textos acadêmicos analisados, de artigos científicos a teses de doutorado, nos quais o conceito de cultura em interações e mediações de Teletandem é discutido. Ao selecionar os trabalhos a serem analisados, buscamos por aqueles que continham o termo "cultura" em seus títulos, conforme demonstrado a seguir:

Quadro 1 - Publicações sobre a questão cultural no contexto do Teletandem.

\begin{tabular}{|c|c|c|}
\hline AUTOR E ANO & NÍVEL & TÍTULO \\
\hline SALOMÃO, A.C.B. (2012) & Tese de Doutorado & $\begin{array}{l}\text { "A cultura e o ensino de língua estrangeira: } \\
\text { Perspectivas para a formação continuada no projeto } \\
\text { Teletandem" }\end{array}$ \\
\hline RODRIGUES, D.G. (2013) & $\begin{array}{l}\text { Dissertação } \\
\text { Mestrado }\end{array}$ & $\begin{array}{l}\text { "A articulação língua-cultura na coconstrução da } \\
\text { competência intercultural em uma parceria de } \\
\text { Teletandem (Português/Espanhol)" }\end{array}$ \\
\hline ANDREU-FUNO, L. (2015) & Tese de Doutorado & $\begin{array}{l}\text { "Teletandem: Um estudo sobre identidades } \\
\text { culturais e sessões de mediação da aprendizagem" }\end{array}$ \\
\hline $\begin{array}{l}\text { TELLES, J.A.; ZAKIR, M.A.; } \\
\text { FUNO, L.B.A. (2015) }\end{array}$ & Artigo científico & "Teletandem e episódios relacionados a cultura" \\
\hline ZAKIR, M.A. (2015) & Tese de Doutorado & $\begin{array}{l}\text { "Cultura e(m) telecolaboração: Uma análise de } \\
\text { parcerias de Teletandem institucional" }\end{array}$ \\
\hline SOUZA, M.G. (2016) & Tese de Doutorado & $\begin{array}{l}\text { "Teletandem e mal-entendidos na comunicação } \\
\text { intercultural online em língua estrangeira" }\end{array}$ \\
\hline
\end{tabular}

Fonte: elaboração própria 
Para a organização do quadro acima, optamos pela ordem cronológica de publicação dos trabalhos, para mostrarmos a sequência temporal destes. Eles serão discutidos em outra ordem, a fim de relacionarmos as reflexões feitas em cada um de acordo com as abordagens adotadas por cada autor acerca das questões culturais identificadas em suas pesquisas.

Iniciaremos nossas observações a partir da tese de doutorado de Andreu-Funo (2015), que tinha como objetivo:

[...] sistematizar um corpo de saberes atuais sobre mediação da aprendizagem em Teletandem e, nesta sistematização, ele contempla uma pautada em dados de pesquisa acerca das relações entre identidades culturais, concepções de cultura e educação para se relacionar com outros povos em contextos virtuais de aprendizagem de línguas (ANDREU-FUNO, 2015, p. 15).

Em seu trabalho, Andreu-Funo (2015) abordou o conceito de cultura considerando o desenvolvimento dos significados do termo ao longo dos anos, desde suas definições iniciais até o amplo leque de significados que ele pode assumir atualmente, passando pelo conceito de transculturalidade proposto por Welsch (1999) até o conceito de dimensões culturais apresentado por Levy (2007). Sobre a transculturalidade, a autora a apresenta, embasada por Welsch (1999), como um complexo emaranhado de culturas modernas que são heterogêneas e englobam várias possibilidades e formas de vida (p. 51). Já em relação às dimensões culturais propostas por Levy (2007), estas são discriminadas como (a) cultura como elemento; (b) cultura como relativa; (c) cultura como filiação a um grupo; (d) cultura como contestada e (e) cultura como individual (variável e múltipla) (ANDREU-FUNO, 2015, p. 52).

Nos resultados obtidos em seu trabalho, após discutir sobre o conceito de mediação e como ela pode contribuir para a experiência dos praticantes de Teletandem, a autora afirma que ações pedagógicas desenvolvidas nas mediações são importantes para a reflexão sobre a relação com outros povos e para a formação docente para o ensino de língua e cultura no século XXI (p. 178). Ela postula que há estereótipos e "mitos" dentro das identidades culturais que concebemos, e que as sessões de mediação podem desestabilizar essas representações essencialistas de identidade cultural.

A autora, em seu trabalho, também buscou identificar quais sentidos são atribuídos à noção de cultura ao longo das sessões de mediação do teletandem, identificando o que ela nomeou de fluxos de singularidade, que salientaram aspectos estáticos, dinâmicos, convergentes e/ou divergentes de cultura. Também observou que 
deles decorreram percepções de cultura que dialogam com as dimensões culturais propostas por Levy (2007) (ANDREU-FUNO, 2015, p. 175)

Telles, Zakir e Funo (2015) também abordaram o conceito das dimensões culturais de Levy (2007) em seu artigo sobre episódios de cultura em contexto de Teletandem. Além de apresentar tais dimensões e também alguns destes episódios, os autores também sustentaram a pressuposição de que os participantes do Teletandem compartilham informações sobre suas culturas, buscando definir o que é aprendizagem cultural, propor uma unidade de análise para interpretação dos dados obtidos e também sugerir diretrizes para futuras pesquisas relacionadas à aprendizagem de línguas e culturas em contextos colaborativos online (TELLES; ZAKIR; FUNO, 2015, p. 359).

Os autores concluem, após analisarem uma interação de Teletandem entre uma brasileira e seu interagente americano, especificamente quando há momentos da conversa em que surge questões relacionadas à cultura, que a compreensão cultural pode ser algo menos fixo e estável (Welsch, 1999), com diferentes dimensões, e dessa forma, é uma maneira mais apropriada para abarcar a complexidade do contexto Teletandem (p. 385-386). Os participantes da pesquisa realizada já possuíam experiências anteriores que influenciaram as expectativas criadas por eles ao começarem a interagir, o que pode ter sido essencial para a forma como eles enxergavam não só sua própria identidade cultural, mas também a do outro.

Zakir (2015) também aborda, em sua tese de doutorado, as dimensões culturais e também o conceito de transculturalidade, que já foi mencionado no trabalho de AndreuFuno (2015). Zakir (2015) buscava investigar o lugar da cultura no contexto de uma parceria de Teletandem, além de identificar concepções de cultura em atividades realizadas pelos participantes da pesquisa em uma plataforma virtual de aprendizagem e compreender de que modo a cultura emerge na dinâmica das interações de Teletandem.

A autora também abordou a cultura no ensino de LE e documentos oficiais, a cultura como discurso, a relativização da cultura nacional no Teletandem, as semelhanças entre culturas e a diversidade cultural presente neste contexto. A partir de suas observações e análises, Zakir (2015) concluiu que o Teletandem desempenha um fundamental papel político-social, assim como pedagógico, para os alunos que entram em contato com os falantes nativos nas interações; as trocas interativas entre pessoas de nacionalidades, culturas e experiências diferentes engendram muitas possibilidades de se compreender a cultura em suas várias dimensões. Ela também afirma que: 
[...] investigar o lugar da cultura no teletandem institucional semiintegrado implica reconhecer, primeiro, que os sujeitos se constituem na relação eu-outro e que cada atividade, cada propósito, cada dimensão espaço-temporal é irrepetível. Pensar o lugar da cultura no teletandem institucional implica pensar na dimensão pedagógica da utilização das interações em teletandem no ensino/aprendizagem de línguas estrangeiras e de língua materna na atualidade. Implica discutir concepções de língua e problematizar o papel de um contexto no qual o contato intercultural online promove mais do que a oportunidade de se praticar uma língua (ZAKIR, 2015, p. 208).

Também focando o contato intercultura, Rodrigues (2013), em sua dissertação de mestrado, buscou investigar o potencial do contexto telecolaborativo Teletandem vistas à aprendizagem intercultural de uma parceria composta por um brasileiro, aprendiz de espanhol como LE, e uma uruguaia, aprendiz de português como "língua adicional”, uma vez que ela fala outras línguas além da nativa. Especificamente, a autora buscou "verificar de que maneira os componentes linguísticos e, principalmente, os culturais são manejados, a fim de promoverem a construção da competência intercultural dos aprendizes" (p. 20). A autora aborda o conceito de interculturalidade como "modos de se compreender o outro e a sua linguagem cultural” (p. 65), e também, segundo Mendes (2007), como uma oportunidade para o incentivo da intercomunicação entre realidades linguístico-culturais diferentes.

A abordagem cultural em seu trabalho consistiu em discutir como os participantes da parceria articulam língua e cultura a fim de promoverem a coconstrução da competência intercultural. A autora, com base em reflexões feitas por Byram (1997) e Meyer (1991), afirma que:

O falante interculturalmente competente é um indivíduo que adquire conhecimento de uma ou mais culturas, identidades sociais e, principalmente, tem a capacidade de lançar mão de recursos a fim de desfrutar da capacidade de descobrir e de se relacionar com pessoas com realidades culturais diferentes da sua (RODRIGUES, 2013, p. 72).

Para aprofundar as reflexões acerca deste fenômeno, Rodrigues (2013) debate os conceitos de teoria sociocultural e Zona de Desenvolvimento Proximal, e também qual é o papel desempenhado pelo outro nas interações, considerando a ajuda mútua (colaboração) no processo de aprendizagem de línguas.

Sobre a teoria sociocultural, a autora, embasada pelos estudos de Vygotsky (1998), afirma que: 
A Teoria Sociocultural se desenvolve em torno de um objeto de estudo que procura reunir em um mesmo modelo explicativo tanto os mecanismos relacionados ao funcionamento psicológico quanto o desenvolvimento do indivíduo inserido em um processo sóciohistórico específico (RODRIGUES, 2013, p. 25).

Ainda seguindo as reflexões de Vygotsky (1998), Rodrigues (2013) aborda a Zona de Desenvolvimento Proximal (ZDP) ao postular que "esta seria a distância entre o que o indivíduo já consegue fazer sem a ajuda do outro e o que ele consegue realizar em colaboração com os outros elementos de seu grupo social” (RODRIGUES, 2013, p. 31). Ela se divide em dois níveis, a saber, o Nível de Desenvolvimento Real e o Nível de Desenvolvimento Potencial, que são referentes às duas capacidades do indivíduo mencionadas anteriormente.

Ao refletir sobre as implicações do outro para a construção do conhecimento, a autora afirma que "a construção de conhecimento, seja em uma língua estrangeira ou em qualquer outro campo do saber, implica em um processo de ação compartilhada" (RODRIGUES, 2013, p. 33), na qual há troca de informações e conflitos de visões distintas.

A partir de suas considerações, a autora concluiu que reflexões de aspectos linguísticos e linguístico-culturais desencadearam a construção da competência intercultural entre os interagentes. Ela também afirma que é importante existir um ambiente telecolaborativo confortável para o desenvolvimento das interações, e que os aspectos históricos, linguísticos e subjetivos da identidade de cada um dos participantes de sua pesquisa puderam fundamentar-se no uso adequado ou não de palavras e expressões das línguas, fato que, para Kramsch (2009), parece ser essencial para a construção de competência comunicativa e cultural (RODRIGUES, 2013, p. 176).

Os conceitos da Zona de Desenvolvimento Proximal também foram abordados por Salomão (2012) em sua tese de doutorado. Os objetivos da autora referentes aos aspectos culturais em contexto de Teletandem buscaram estudar as concepções de cultura dos professores brasileiros, suas crenças sobre a cultura-língua do outro e a contribuição de um curso de formação continuada para professores de língua espanhola da rede pública, promovido pelos coordenadores e pesquisadores do projeto Teletandem. A autora também aborda a relação entre língua e cultura no ensino de línguas, a cultura no conceito de competência comunicativa, os componentes da 
competência sociocultural e as abordagens para ensino de cultura na educação linguística.

Com base em sua análise, Salomão (2012) concluiu que as concepções de cultura dos professores pesquisados podem ser resquício de uma visão modernista de cultura no ensino de línguas e que as crenças levantadas sobre os blocos culturais geralmente se apresentaram na forma de estereótipos. As crenças dos professores estavam intimamente relacionadas à sua concepção de cultura como informação e práticas, profundamente associadas a status de superioridade e inferioridade atribuídos pelos professores a determinadas culturas, e relacionadas ao ensino na forma de diferenças que "valem a pena aprender".

Além disso, Salomão (2012) também afirma que é necessário problematizar no teletandem e na educação de professores a crença do falante nativo como informante legítimo e ideal de língua e cultura por natureza, de modo a descobrir a maneira como as concepções de cultura interagem com a crença de muitos professores sobre a necessidade da experiência in loco para legitimação do conhecimento sobre língua e cultura (p. 242). Para a autora, devem ser criadas estratégias de ensino específicas, que não sejam eventuais nem aleatórias, para o desenvolvimento cognitivo com uso das interações, pois elas trazem a oportunidade de vivenciar dialogicamente experiências que servirão para reformulação de concepções e crenças por meio da interação entre conceitos cotidianos e científicos (SALOMÃO, 2012, p. 245).

As características de interações interculturais de aprendizagem, a comunicação intercultural e a interação intercultural no Teletandem também foram abordadas por Souza (2016) em sua tese de doutorado. Os objetivos de seu trabalho eram: (a) analisar as características do diálogo intercultural em teletandem, por meio da mobilização dos conhecimentos dos interagentes e pela perspectiva linguística e sócio-discursiva dos processos de negociação de sentidos; e (b) identificar os mal-entendidos ocorridos ou possíveis nas interações (SOUZA, 2016, p. 28).

Conforme analisado pela autora, a emergência dos mal-entendidos pode ocorrer tanto por meio de choques linguísticos quanto por meio de choques culturais. Os choques linguísticos são mais frequentes quando não há muito domínio da língua estrangeira pelos falantes, e os choques culturais, quando os interagentes percebem que o outro idioma não funciona sob as mesmas estratégias aplicadas na língua materna. Considerando estes fenômenos, a autora postula que: 
O choque linguístico-cultural, portanto, relaciona-se ao processo de aprendizagem de modo mais evidente e pontual, pois há uma indicação explícita, percepção ou sensação entre os aprendizes de sua ocorrência. Tal fenômeno dialoga com e pode ocasionar situações de mal-entendido. No entanto, este último compreende uma maior complexidade, já que nem sempre é percebido e declarado em uma situação comunicativa. (SOUZA, 2016, p. 47-48)

Entretanto, esses mal-entendidos nem sempre devem ser vistos como algo negativo e provocador de frustrações, pois ainda de acordo com a autora, é por meio deles que ocorrem as negociações de sentido de modo colaborativo e a contribuição para a aprendizagem dos envolvidos na interação. Mesmo em situações que a princípio pareçam constrangedoras, os interagentes podem extrair conhecimento e aperfeiçoar o domínio da língua-alvo por meio desses mal-entendidos, desde que bem administrados colaborativamente por ambas as partes.

O choque linguístico-cultural também pode ser benéfico ao provocar os interagentes a refletirem sobre diversos aspectos da língua e cultura do outro e também de suas próprias, mais um elemento essencial para o desenvolvimento da competência intercultural e também do aprimoramento da experiência no Teletandem.

A autora também observou, nos dados coletados nas interações que fizeram parte de sua pesquisa, que houve compartilhamento de ideias sedimentadas e generalizações, em se tratando de aspectos culturais. Também foram identificadas diferenças que se refletem nos modos como cada interagente representa e constrói suas culturas nacionais discursivamente, ressaltando aspectos generalizantes positivos ou negativos (SOUZA, 2013, p. 143).

A dimensão cultural se mostrou permeada nos discursos dos interagentes e impactou os processos de negociação de sentidos, e foi possível observar também a recorrência dos discursos reducionistas sobre culturas nacionais. A autora também observou a existência de uma superficialidade das representações discursivas dos interagentes sobre suas culturas nacionais, o que demonstra a limitação inerente ao conceito tradicional de cultura como uma esfera independente. Identificou-se, também, que "a ausência de negociações mais plurais e diversificadas também corrobora para a configuração de diálogos limitadores, permeados por representações ideologicamente alinhadas ao status quo" (SOUZA, 2013, p. 149).

Com base nas observações que fizemos acerca dos trabalhos acadêmicos aqui contemplados, encaminhamos nosso texto para suas considerações finais, abordando os 
aspectos discutidos até então e comparando-os a fim de observar semelhanças e diferenças entre a abordagem cultural realizada nas publicações supracitadas.

\section{Considerações finais}

Para a elaboração deste artigo, buscamos refletir sobre como o componente cultural está presente no contexto de Teletandem, tanto na experiência dos participantes, durante as interações, quanto nas sessões de mediação. Para isso, tomamos como base textos publicados anteriormente acerca deste tema, que variaram desde artigos científicos até teses de doutorado.

Ao considerarmos as reflexões feitas pelos autores, buscamos fazer comparações entre os trabalhos aqui referenciados, que nos mostraram que os conceitos de cultura usados foram adequados a propósitos diferentes, de acordo com o enfoque e objetivos de cada investigação. Pudemos identificar que os conceitos de competência comunicativa e cultura como discurso foram citados, além de outros conceitos, como a transculturalidade, as dimensões culturais e a interculturalidade, seguindo os pressupostos teóricos de autores como Kramsch (2009, 2011), Welsch (1999), Levy (2007) e Mendes (2007), respectivamente.

Além disso, também observamos que todos os trabalhos debatem sobre a complexidade da interação entre o "eu" e o "outro" no contexto do teletandem, e como a cultura é vista em diferentes dimensões nesse contexto telecolaborativo. A experiência vivida pelos interagentes assemelha-se a uma "imersão" no contexto do outro, considerando seus aspectos culturais, sociais e linguísticos.

De modo geral, pudemos observar que, independentemente dos objetivos das pesquisas, os conceitos de cultura parecem bastante próximos, principalmente devido ao uso de autores semelhantes. Também se observou a necessidade da criação de estratégias, por meio de mecanismos ou diretrizes, para a realização das sessões de mediação, a fim de estabilizar as representações essencialistas da identidade cultural que surgem durante sessões de teletandem e também nas mediações. Os trabalhos apontam para a necessidade de se olhar para a maneira como aspectos culturais são abordados pelos interagentes durante suas sessões de teletandem, considerando como eles enxergam a cultura própria e também a do outro.

Assim, em se tratando de um contexto telecolaborativo que traz à tona a possibilidade de um encontro com o outro, o Teletandem promove de situações de 
interação com vieses culturais que podem ser observados e analisados a partir de diferentes perspectivas. Podemos afirmar que as pesquisas analisadas trazem à luz reflexões variadas a respeito do componente cultural nas interações de Teletandem, mas coadunam que essa modalidade de aprendizagem colaborativa promove a emergência do componente cultural de forma dinâmica entre os participantes, por meio de um processo constitutivo de reflexão sobre sua própria língua e cultura proporcionada pela interação com o outro.

\section{REFERÊNCIAS}

ANDREU-FUNO, L. Teletandem: Um estudo sobre identidades culturais e sessões de mediação da aprendizagem. Tese de Doutorado. P.P.G. em Estudos Linguísticos, UNESP - Universidade Estadual Paulista, 2015.

BRAMMERTS, H. Language Learning in Tandem Using the Internet. In:

WARSCHAUER, M. (org.) Telecollaboration in foreign language learning. Manoa: University of Hawai'i Press, 1996.

BYRAM, M. Teaching and Assessing Intercultural Communicative Competence. Clevedon: Multilingual Matters, 1997.

EVANGELISTA, M. C.; SALOMÃO, A. C. Mediation in Teletandem.

Pandaemonium Germanicum, v. 22, n. 36, p. 153-177, 2019.

KRAMSCH, C. Cultural perspectives on language learning and teaching. In: KNAPP, $\mathrm{K}$; SEIDLHOFER, B. (Eds.). Handbook of foreign language communication and learning. Berlin: Mouton de Gruyter, 2009. p. 219-245.

KRAMSCH, C. The symbolic dimensions of the intercultural. Language and Teaching, v. 44, n. 3, p. 354-367, 2011.

LEVY, M. Culture, Culture Learning and New Technologies: Towards a pedagogical framework. Language Learning \& Technology, v. 11, n. 2, p. 104-127, 2017.

MENDES, E. Perspectivas interculturais no ensino de línguas: uma relação "entreculturas". In: ALVAREZ, M. L. O; SILVA, K. A. da. (Org.). Linguística Aplicada: múltiplos olhares. Brasília: Pontes, p.119-139, 2007.

MEYER, M. Developing transcultural competence: case studies of advanced foreign language learners. In: BUTJES, D. BYRAM, M. (Org.) Developing languages and cultures. 1991.

OLIVEIRA, A. P. Tips for Teaching Culture in a Globalized World. In: LIMA, D. C. (Ed.). Language and its cultural substrate: perspectives for a globalized world. Campinas: Pontes, 2012. p. 83-107. 
SALOMÃO, A. C. B. Vizinhança global ou proximidade imposta? Impactos da comunicação intercultural mediada por computador sobre o papel da cultura no ensino de língua inglesa. D.E.L.T.A., São Paulo, v. 27, n. 2, p. 235-256, 2011.

SALOMÃO, A. C. B. A cultura e o ensino de língua estrangeira: Perspectivas para a formação continuada no projeto teletandem. Tese de Doutorado. P.P.G. em Estudos Linguísticos, UNESP - Universidade Estadual Paulista, 2012.

SALOMÃO, A. C. B. O componente cultural no ensino e aprendizagem de línguas: desenvolvimento histórico e perspectivas na contemporaneidade. Trabalhos em Linguística Aplicada, Campinas, v. 54, n. 2, p. 361-392, jul./set. 2015.

SOUZA, M. G. Teletandem e mal-entendidos na comunicação intercultural online em língua estrangeira. Tese de Doutorado. P.P.G. em Estudos Linguísticos, UNESP Universidade Estadual Paulista, 2016.

RODRIGUES, D. G. A articulação língua-cultura na coconstrução da competência intercultural em uma parceria de Teletandem (Português/Espanhol). Dissertação de Mestrado. UNESP, P.P.G. em Estudos Linguísticos. Orientadora: Dra. Ana Mariza Benedetti, 2013.

TELLES, J. A.; VASSALLO, M. L. A. Foreign language learning in-tandem: Theoretical principles and research perspectives. The ESPecialist, v. 27, n. 1, p. 83$118,2006$.

TELLES, J. A.; ZAKIR, M. A.; FUNO, L. B. A. Teletandem e episódios relacionados a cultura. D.E.L.T.A. - Revista de Documentação e Estudos em Linguística Teórica e Aplicada, v. 31, n. 2, p. 359-389, 2015.

VASSALLO, M. L.; TELLES, J. A. Foreign language learning in-tandem: Teletandem as an alternative proposal in CALLT. The ESPecialist, v. 27, n. 2, p. 189-212, 2006.

VYGOTSKY, L. A formação social da mente. Trad. José Cipolla Neto; Luis Silveira Menna Barreto; Solange Castro Afeche. São Paulo: Martins Fontes, 1998.

WELSCH, W. Transculturality - the Puzzling Form of Cultures Today. In: Spaces of Culture: City, Nation, World, ed. by Mike Featherstone and Scott Lash, London: Sage 1999, 194-213.

ZAKIR, M. A. Cultura e(m) telecolaboração: Uma análise de parcerias de teletandem institucional. Tese de Doutorado. P.P.G. em Estudos Linguísticos, UNESP Universidade Estadual Paulista, 2015. 


\section{Como referenciar este artigo}

MORETTI, G.; SALOMÃO, A. C. B. Os componentes culturais nas interações do Teletandem: teorias e reflexões. Rev. EntreLínguas, Araraquara, v. 5, n. 2, p. 378-393, jul./dez. 2019. E-ISSN: 2447-3529. DOI: 10.29051/el.v5i2.12746

Submetido em: 01/03/2019

Revisões requeridas: $10 / 05 / 2019$

Aprovado em: 28/06/2019

Publicado em: 01/10/2019 